\title{
Structure of Aerodynamic Noise Sources Generated in Production Vehicle*
}

\author{
Takahide NOUZAWA**, Ye LI**, Naohiko KASAKI** \\ and Takaki NAKAMURA** \\ ${ }^{* *}$ Mazda Motor Corporation \\ Shinchi 3-1, Fuchu-cho, Aki-gun, Hiroshima, Japan \\ E-mail: nouzawa.t@mazda.co.jp
}

\begin{abstract}
Experiment has been performed to clarify the structure of aerodynamic noise sources generated in a production vehicle. The details of noise sources were measured from the outside and inside of vehicle by applying noise source identification techniques. The noise sources show high level in the regions of bonnet head, front underbody, side window and rear window. Moreover, the noise sources are dominated by that from underbody in the low frequency, and side window in the high frequency, respectively. The velocity dependency of aerodynamic noise was analyzed based on Lighthill-Curle analogy. As a result, it is found that the noise sources around vehicle are mainly dipole sources.
\end{abstract}

Key words: Aerodynamic Noise, External Flow, Flow Visualization, Vehicle

\section{Introduction}

The reduction of wind noise becomes more and more important for the comfortable vehicle development, because the noise caused by engine, power train, tires, and other noise sources has been steadily reduced in recent years. The wind noise can be divided into leak noise and aerodynamic noise according to its generation mechanism. Among these, the aerodynamic noise is closely related to the complex unsteady flow around vehicle, so it is very difficult to clarify the generation mechanism of aerodynamic noise. In the case of production vehicle, the noise source is mainly induced by the flow separation around body. Many researchers ${ }^{(1)(2)}$ have revealed that strong aerodynamic noise is generated by the flow separation around the regions of front pillar and sideview mirror. In addition, the other main contributor to aerodynamic noise is coming from the underbody ${ }^{(3)}$. However, the knowledge about the whole distribution of wind noise sources is still insufficient, and the relation between the noise source and flow field is not sufficiently clarified.

In this study, the wind noise sources were measured by applying noise source identification equipments from the outside and inside of vehicle. Then the whole structure of aerodynamic noise sources was clarified, especially the noise sources from side window and underbody were investigated. Moreover, the relation between the noise source and interior noise was analyzed by applying Lighthill-Curle's acoustic analogy.

\section{Nomenclature}

$$
\begin{array}{ll}
I & \text { : sound intensity, }\left[\mathrm{W} / \mathrm{m}^{2}\right] \\
N & \text { : multiplier proportional to sound intensity } I \text { and vehicle speed } U \\
p_{0} & \text { : reference pressure of } \mathrm{SPL}(\mathrm{dB})=2 * 10^{-5}, \mathrm{~Pa}
\end{array}
$$

Copyright $\odot 2011$ by JSME 
$p \quad$ : sound pressure, $\mathrm{Pa}$

$S P L$ : sound pressure level, $\mathrm{dB}$

$t \quad$ : time, $s$

$U \quad$ : vehicle speed, $\mathrm{m} / \mathrm{s}$

\section{Experimental Method}

The experiments were conducted in the large-scale low-noise wind tunnel of Railway Technical Research Institute (RTRI). The main feature of this wind tunnel is its low background noise level $(55 \mathrm{~dB}$ in wind speed of $38.9 \mathrm{~m} / \mathrm{s})$, so the aerodynamic noise can be measured at high precision. The open-type nozzle of $5 \mathrm{~m}$ (width) and $3 \mathrm{~m}$ (height) was used to measure the aerodynamic noise sources from the outside of vehicle. The vehicle used for this study was a 5-doors hatchback production vehicle shown in Fig. 1. In order to investigate the effect of underbody noise source, the underbody-closed model (Fig. 1(b)) that closed entire airflow to the underbody was applied in addition to the original model (Fig. 1(a)). The wind tunnel experiment with moving belt is generally conducted to investigate the underbody flow. However, the purpose of present study is to clarify the whole structure of aerodynamic noise sources more than the detailed distribution of noise source in underbody. So the experiments were conducted in the conditions with fixed ground floor and without tire rotation, which can remove sound sources other than aerodynamic noise.

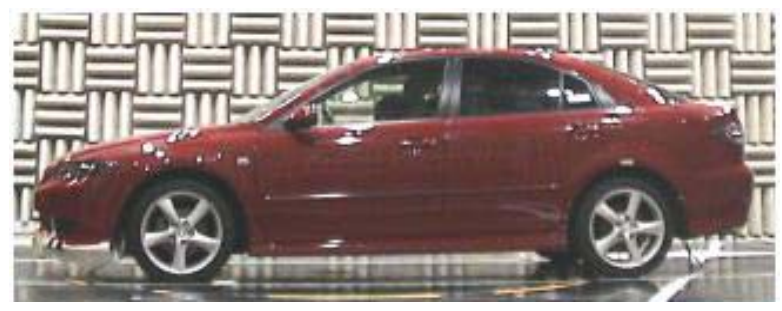

(a) Original Model

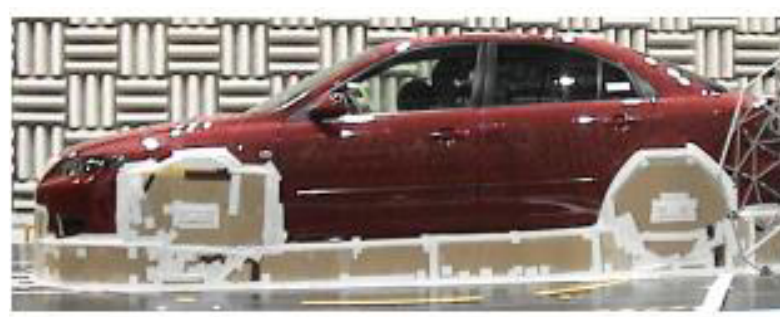

(b) Underbody-closed Model

Fig.1 Testing models used in current study

The external noise source was measured by means of PULSE Beamforming ${ }^{(4)}$ produced by Brüel \& Kjær. The 66-channel Beamforming Wheel Array (abbreviated as Beamforming Array hereafter) was applied and the diameter of array is $1 \mathrm{~m}$. The resolutions of noise source at $1 \mathrm{~m}$ are $68 \mathrm{~cm}$ for $500 \mathrm{~Hz}, 34 \mathrm{~cm}$ for $1 \mathrm{kHz}$, respectively. Because the vehicle size is quite large compared with the array, the noise source area was divided into four, and measured separately. For each time, the array was placed in the different locations, which are named as Front upper side, Rear upper side, Front lateral side and Rear lateral side. The detailed Beamforming Array's position is shown in Fig. 2. Only the results measured from the Front upper side and Front lateral side are described in this paper due to space limitation. 


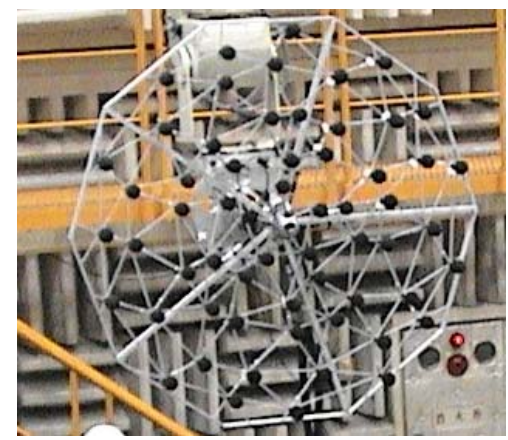

(a) Beamforming wheel array

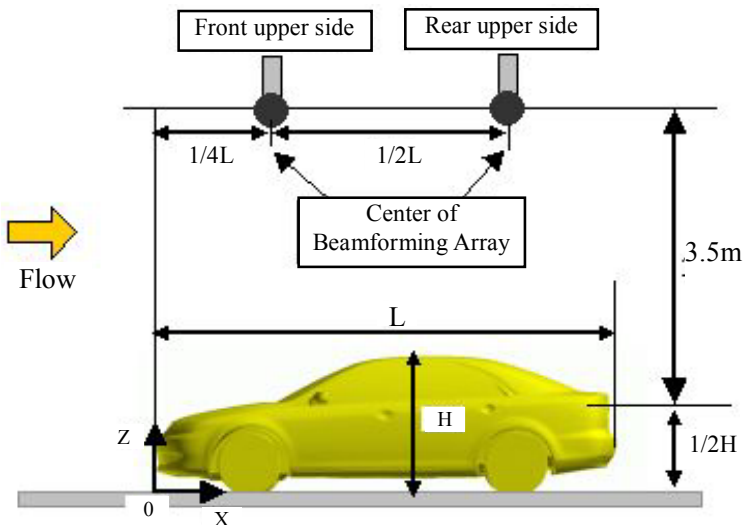

(b) Array's positions in the upper side

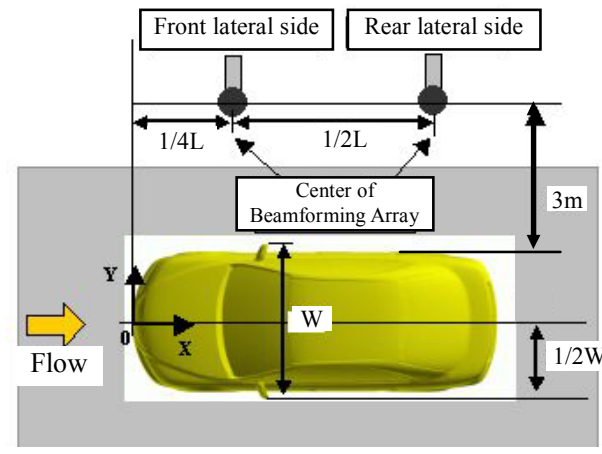

(c) Array's positions in the lateral side

Fig.2 Beamforming array and its measurement positions

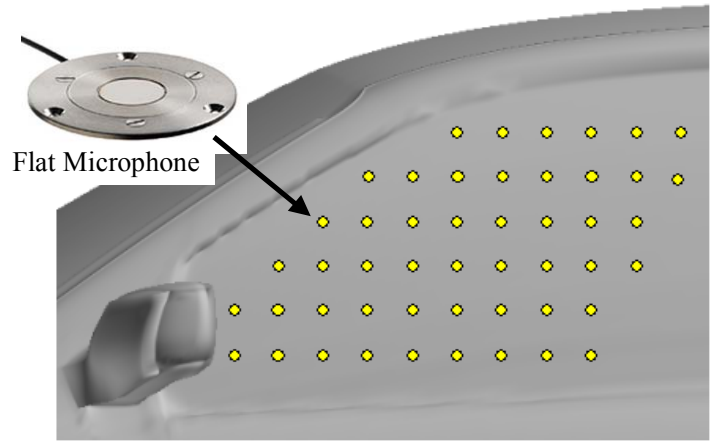

Fig.3 Flat microphones mounted on side window 


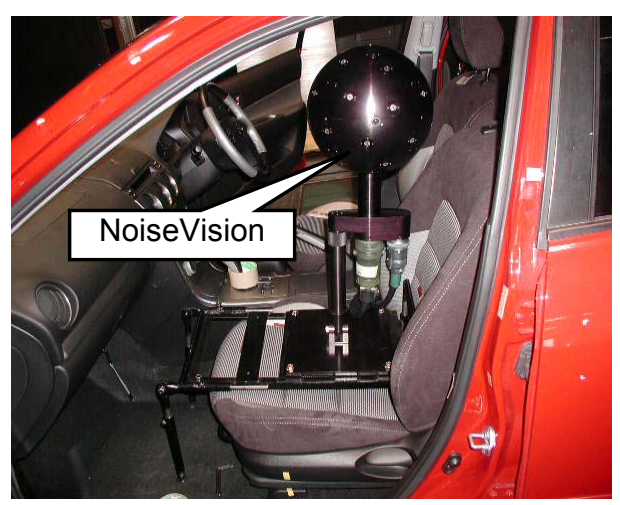

Fig.4 NoiseVision used to identify the internal noise sources

The distance between the center of array and vehicle is $3.5 \mathrm{~m}$ and $3 \mathrm{~m}$ for the upper side and lateral side, respectively. The array was positioned in the outside of the flow field, such that the airflow over the microphones was insignificant. Therefore, these microphones mounted on the array can accurately capture the sound radiated from the vehicle.

Beamforming can analyze the whole image of the aerodynamic noise around vehicle, but only the two dimensional information about the location and intensity of sound source is obtained. Then, the flat microphones (B\&K 4948, Fig. 3) were mounted on the surface of side window to measure the surface pressure fluctuation induced by the flow separation in front pillar and sideview mirror. Since the thickness of microphone is only $2.5 \mathrm{~mm}$, the influence of microphone on flow field can be ignored. The surface pressure fluctuation of 98 points in total were measured on the side window. In addition, the wind noise source was also investigated from the inside of vehicle by applying the noise source identification apparatus 'NoiseVision' (Fig. 4). The device has 31 microphones and 11 CCD cameras, which can analyze the noise source in closed space. The noise source distribution analyzed by microphones can be superimposed on the photos taken by CCD cameras to capture the accurate position of noise source. Moreover, the interior noise was evaluated using the Dummy Head which can reproduce a human ear.

All the testing results described in this paper were obtained under the condition with zero yaw angle. To clarify the dependency of sound on the vehicle speed, three vehicle speeds: $27.8 \mathrm{~m} / \mathrm{s}, 38.9 \mathrm{~m} / \mathrm{s}$, and $50 \mathrm{~m} / \mathrm{s}$ were measured. The experiment results of $38.9 \mathrm{~m} / \mathrm{s}$ are shown in this paper, except when the vehicle speed dependency of sound is described.

\section{Results and Discussion}

\subsection{Whole Image of Aerodynamic Noise Source Around Vehicle}

Firstly, Fig. 5 shows the distribution of external noise sources measured by Beamforming Array from the outside of vehicle in the case of Original Model, where the results in $1 / 3$ Octave center frequency of $500 \mathrm{~Hz}, 1 \mathrm{kHz}, 2 \mathrm{kHz}$, and $4 \mathrm{kHz}$ are shown. The photograph of vehicle and the distribution figure of noise source show same area, so the position of noise source can be analyzed by superimposing these figures. In the results measured from the upper side of vehicle (Fig. 5(a)), it can be seen that the noise source from the bonnet head (A region) is dominant in the frequencies of $1 \mathrm{kHz}$ and $2 \mathrm{kHz}$. With the increase of frequency, the noise source from bonnet head decreases, however that from the front pillar and sideview mirror (B region) becomes strong. A slightly strong noise source is also observed from the roof end in the frequency over $1 \mathrm{kHz}$, but these results are omitted in this paper. 

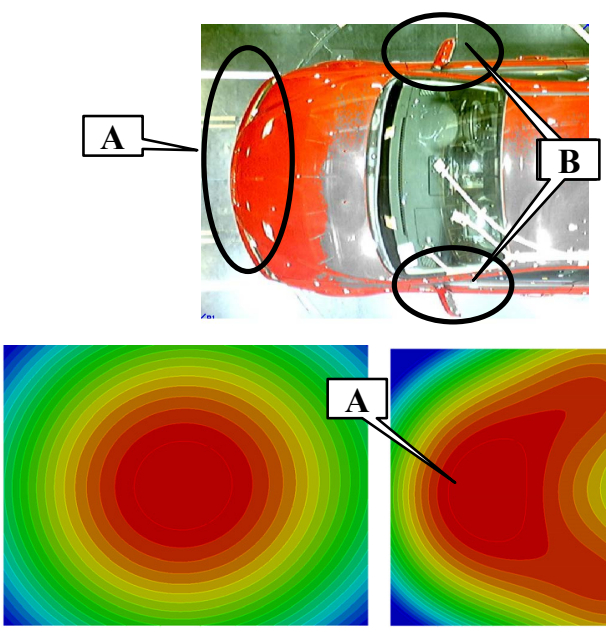

$500 \mathrm{~Hz}(64 \mathrm{~dB}-66.5 \mathrm{~dB})$

$1 \mathrm{KHz}(54 \mathrm{~dB}-57.5 \mathrm{~dB})$

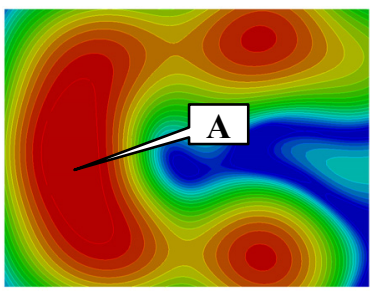

$2 \mathrm{KHz}(35 \mathrm{~dB}-50 \mathrm{~dB})$

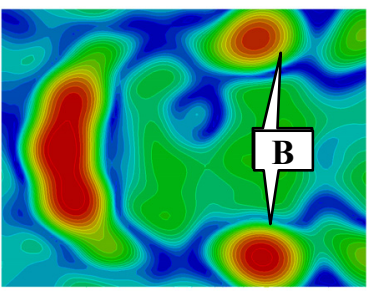

$4 \mathrm{KHz}(30 \mathrm{~dB}-45 \mathrm{~dB})$ Low High

(a) Noise sources measured from upper side

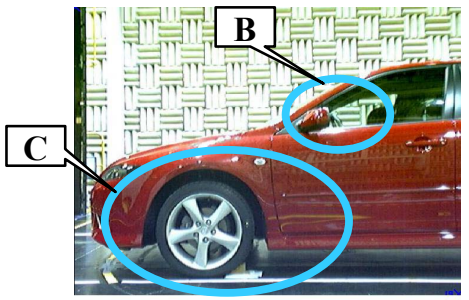

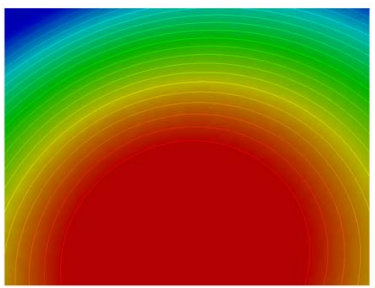

$500 \mathrm{~Hz}(65 \mathrm{~dB}-70 \mathrm{~dB})$

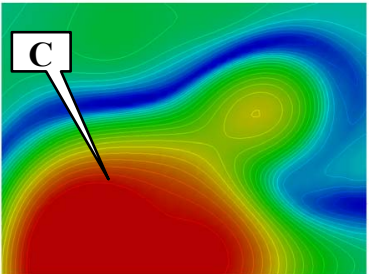

$2 \mathrm{KHz}(40 \mathrm{~dB}-55 \mathrm{~dB})$

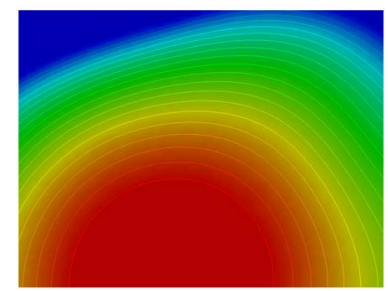

$1 \mathrm{KHz}(50 \mathrm{~dB}-65 \mathrm{~dB})$

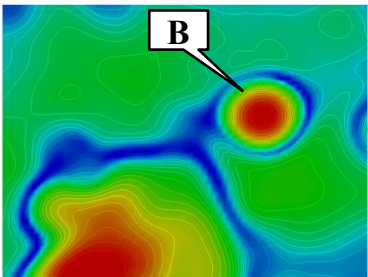

$4 \mathrm{KHz}(35 \mathrm{~dB}-50 \mathrm{~dB})$

Low

High

(b) Noise sources measured from lateral side

Fig.5 Distribution of aerodynamic noise source measured by Beamforming Array (Original Model) 
From the noise source distribution measured from the lateral side (Fig. 5(b)), we can see that the noise source in the front underbody ( $\mathrm{C}$ region) is significantly strong in all frequency range. Especially in the low frequency less than $1 \mathrm{kHz}$, the noise source from the underbody is dominant. However, in the high frequency more than $2 \mathrm{kHz}$, the strong noise source (B region) is observed in the surrounding of side window, which corresponds to the results measured from the upper side. From above measurement results, it is summarized that the aerodynamic noise around vehicle is mainly generated from the regions near bonnet head, side window, roof end, and front underbody.

The aerodynamic noise source generated around vehicle can be basically divided into two types. One is due to the flow turbulence occurred in the space around vehicle, and another is generated by the pressure fluctuation on the body surface of vehicle. According to Lighthill-Curle's acoustic analogy ${ }^{(5)(6)}$, the noise source induced by the flow turbulence in space is quadrupole, and the intensity of sound $I$ in the far field is proportional to the $8^{\text {th }}$ power of the vehicle speed $U\left(I \propto U^{8}\right)$. On the other hand, the noise source by the pressure fluctuation of body surface is dipole, and the intensity of sound in the far field is proportional to the $6^{\text {th }}$ power of the speed $\left(I \propto U^{6}\right)$.

To clarify these two kinds of noise sources in vehicle, the multiplier relation between the vehicle speed and the far-field sound was investigated. Fig. 7 shows the sound pressure level (SPL) measured at the position (Fig. 6) that is $3 \mathrm{~m}$ away from the body side. It can be seen that the sound pressure rises greatly as the vehicle speed goes up. However, the sound pressure shows a peak in $630 \mathrm{~Hz} \sim 1 \mathrm{kHz}$ regardless of vehicle speed, and the pattern of sound pressure is similar.

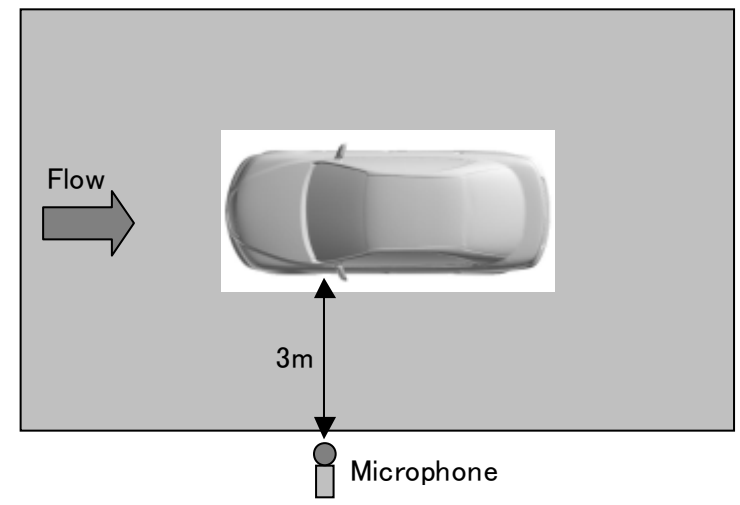

Fig.6 Location of microphone used to measure the exterior noise

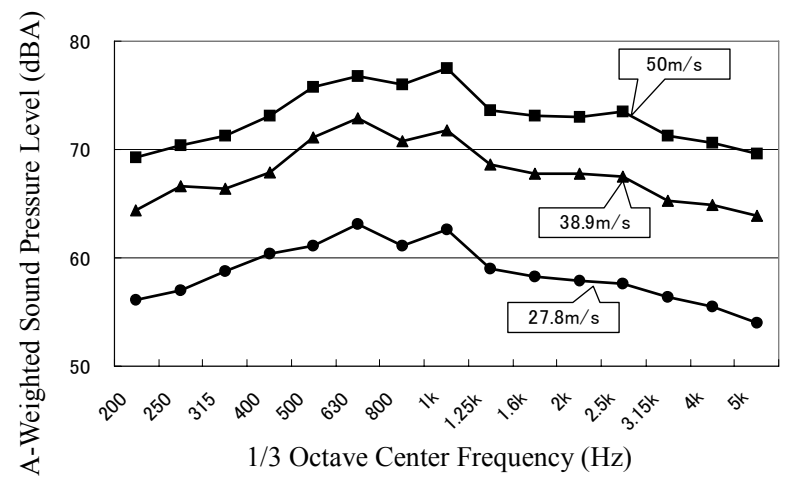

Fig. 7 SPL of exterior noise measured in the outside of vehicle (Original Model) 
It is assumed that the relation between sound intensity $I$ and vehicle speed $U$ can be expressed as follows.

$$
I \propto U^{N}
$$

Moreover, the relation between sound intensity $I$, sound pressure $p$, and sound pressure level $S P L$ can be expressed as follows.

$$
I \propto p^{2}
$$

$$
S P L=20 \log \left(p / p_{0}\right)
$$

From the above expressions (1) (3), the relation between the sound pressure level $S P L$ and vehicle speed $U$ can be derived as follows.

$$
S P L=10 * N * \log (U)+C
$$

Fig. 8 shows the result of multiplier $N$ that is obtained by applying the vehicle speed $U$ and sound pressure level SPL shown in Fig. 7. The multiplier $N$ is about 6.5 in the frequency over $500 \mathrm{~Hz}$. It means that the sound intensity is proportional to about $6.5^{\text {th }}$ power of vehicle speed. It is well known that the sound generated from quadruple noise source is proportional to the $8^{\text {th }}$ power of the vehicle speed, and sound from dipole source is proportional to the $6^{\text {th }}$ power to the speed. Moreover, it has been shown that the flow separation from front pillar interferes with the shear layer separated behind sideview mirror in vehicle ${ }^{(1)}$. The spatial and temporal variation of vorticity can be observed from the flow field, which implies the existence of quadrupole noise source. However, from the results in Fig.8, it can be seen that the noise source around vehicle is dominated by the dipole source in comparison with quadrupole source. It means that the main noise source of aerodynamic noise generated around vehicle is the dipole noise source induced by the pressure fluctuation on the surface of body. In addition, it can be seen from Fig. 7 that the peak frequency of spectrum is almost same even if the vehicle speed changes. It indicates that the flow pattern around vehicle does not vary with the vehicle speed, and only the level of pressure fluctuation becomes strong.

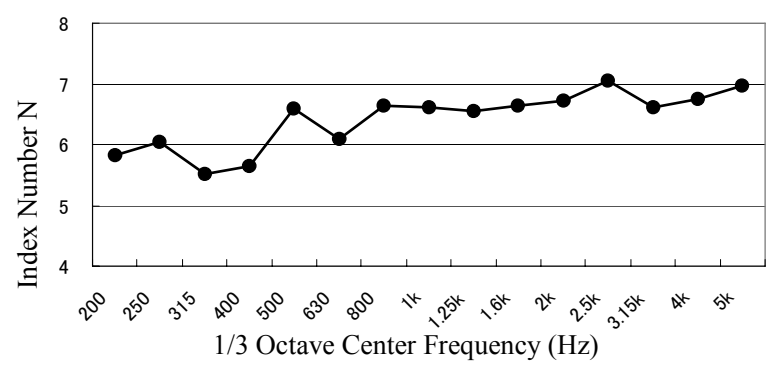

Fig. 8 Velocity dependency of exterior noise (Original Model)

\subsection{Aerodynamic Noise Source Near Side Window}

From the results of noise source measured from the outside of vehicle shown in Fig.5, the strong noise source is observed from the side window direction in the frequency over $1 \mathrm{kHz}$. According to Lighthill-Curle's acoustic analogy, the sound pressure generated from the compact noise source can be predicted by using following formula.

$$
p(\overrightarrow{\mathbf{x}}, t)=\frac{1}{4 \pi c} \int_{s} \frac{\overrightarrow{\mathbf{n}} \cdot \overrightarrow{\mathbf{r}}}{r^{2}} \frac{\partial p}{\partial t} d s
$$

It demonstrates that the time variation of pressure fluctuation on the body surface plays 
an important role on the generation of aerodynamic noise. Fig. 9 shows the time differentiation of pressure fluctuation $d p / d t$ measured on the surface of side window by applying flat microphones shown in Fig. 3, where the distribution of overall value from $500 \mathrm{~Hz}$ to $8 \mathrm{kHz}$ is plotted. The time differentiation of pressure fluctuation is large in the region behind front pillar and sideview mirror, which suggests that strong aerodynamic noise is generated from this region. From the results of flow field obtained by CFD and experiment, it has been shown that the flow separation vortex from front pillar interferes with the flow separated behind sideview mirror in this region ${ }^{(1)}$. Therefore, these flow separation vortices cause the pressure fluctuation that corresponds to noise source. To reduce the aerodynamic noise near side window, it is necessary to optimize the shape of front pillar and sideview mirror in order to inhibit the flow separation.

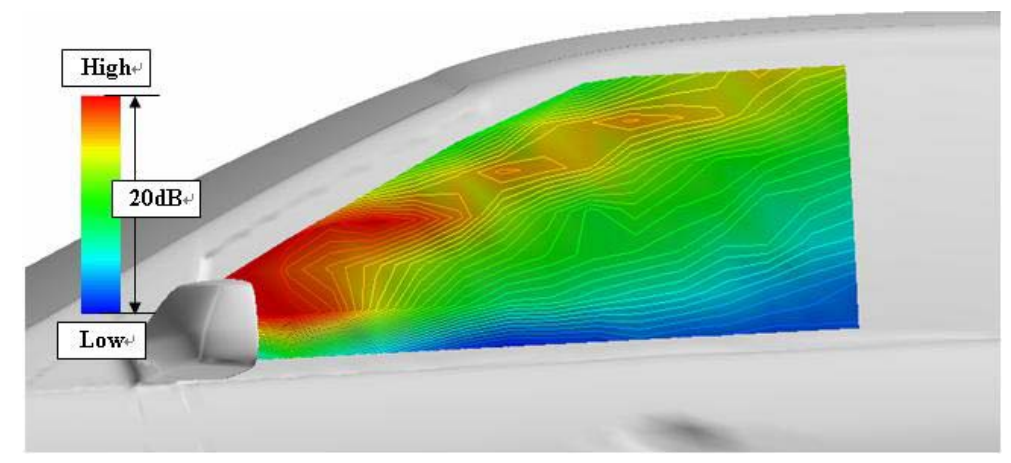

Fig. 9 Time differentiation of surface pressure fluctuation $d p / d t$ (Original Model)

\subsection{Aerodynamic Noise Source in Underbody}

From the noise source distribution shown in Fig. 5(b), we can see that the noise source coming from the direction of front underbody is dominant in the frequency below $1 \mathrm{kHz}$. To verify whether these noise sources are really generated by the underbody flow, Beamforming Array was applied to measure the noise source for the underbody-closed model. The measured results are shown in Fig. 10, where the color range in graph is same as that in Fig. 5. Compared with the noise source distribution (Fig. 5) in the model with underbody flow, the noise source in the underbody ( $\mathrm{C}$ region) becomes significantly small. In particular, the noise source is not almost observed in the underbody for the frequency below $1 \mathrm{kHz}$. These results show that strong noise source is generated from the flow of underbody.

In order to clarify how the noise source from underbody affects the interior noise, Fig. 11 shows the sound pressure level measured at the position of driver's ears in the original model and underbody-closed model. The interior noise is reduced $5 \mathrm{~dB}$ at the maximum in the low frequency below $630 \mathrm{~Hz}$ when the noise source in underbody disappears. It denotes that the noise source in underbody greatly contributes the generation of interior noise in low frequency. Meanwhile, the sound pressure slightly increases from $1 \mathrm{kHz}$ to $4 \mathrm{kHz}$ in the underbody-closed model. The reason for this increase of sound pressure can be explained as follows: By shutting the flow to the underbody, the flux to the vehicle upper side increases. Therefore, the flow separation in the front pillar and sideview mirror becomes strong, which strengthens the noise source (B region in Fig. 10) near the side window. 


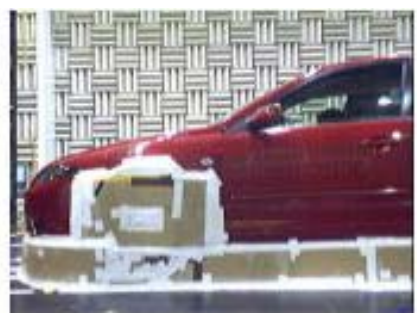

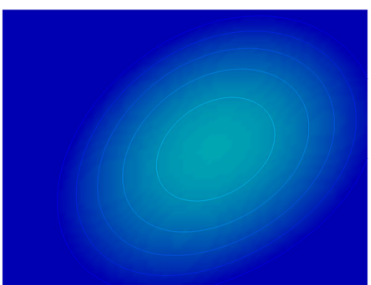

$500 \mathrm{~Hz}(65 \mathrm{~dB}-70 \mathrm{~dB})$

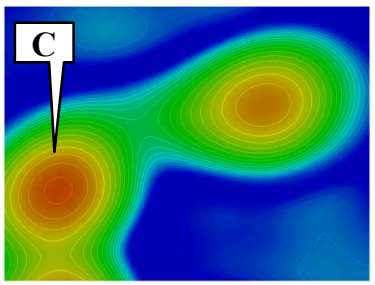

$2 \mathrm{KHz}(40 \mathrm{~dB}-55 \mathrm{~dB})$

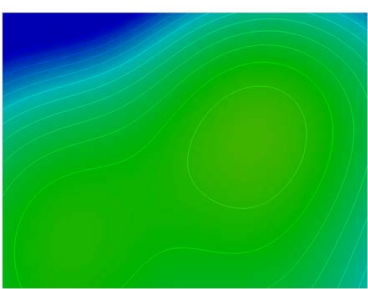

$1 \mathrm{KHz}(50 \mathrm{~dB}-65 \mathrm{~dB})$

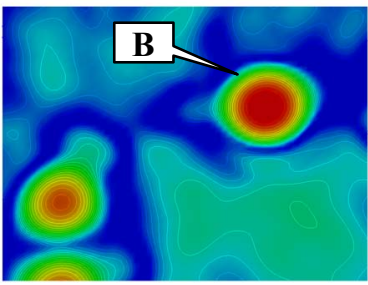

$4 \mathrm{KHz}(35 \mathrm{~dB}-50 \mathrm{~dB})$

Low

Fig.10 Distribution of external wind noise sources measured from front lateral side (Underbody-closed Model)

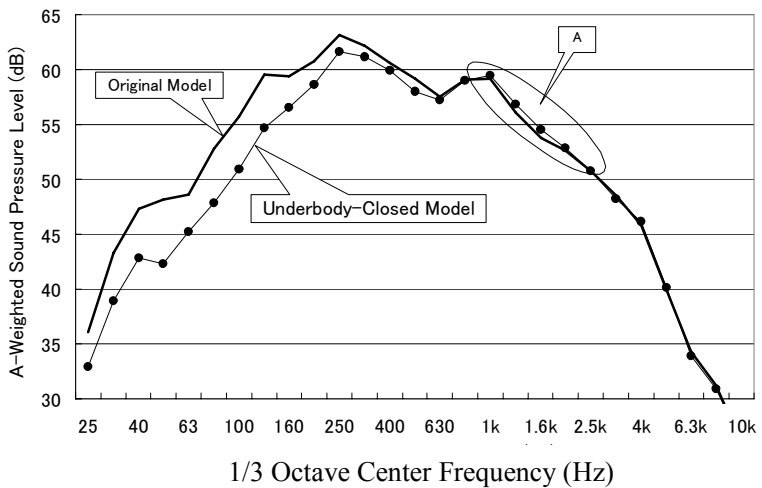

Fig.11 Comparison of A-weighted interior noise between original model and underbody-closed model

\subsection{Aerodynamic Noise Source Measured From Inside of Vehicle}

To investigate how the aerodynamic noise generated in the outside of vehicle is heard in the cabin, Fig. 12 shows the distribution of noise source measured by applying NoiseVision from the inside of vehicle. In the frequencies of $500 \mathrm{~Hz}$ and $1 \mathrm{kHz}$, strong noise source can be observed near the lower part of front pillar and sideview mirror. However, the noise source coming from the mirror decreases, and strong noise source is generated from the whole front pillar in $2 \mathrm{kHz}$. These noise sources are corresponding to the noise sources near side window ( B region in Fig.5 and Fig.9) measured from the outside of vehicle. That is, the aerodynamic noise generated in the outside of vehicle transmits into the cabin through the front pillar and side window glass. Therefore, to reduce the wind noise in the cabin, it is important to not only inhibit the noise source in the outside of vehicle, but also strengthen the insulation performance around front pillar and side window. 


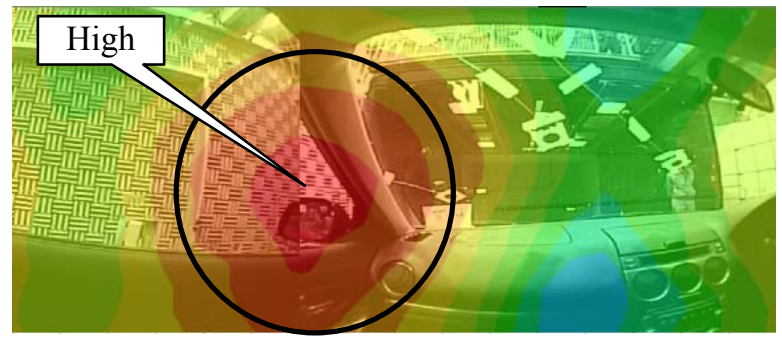

$500 \mathrm{~Hz}(109 \mathrm{~dB}-116 \mathrm{~dB})$
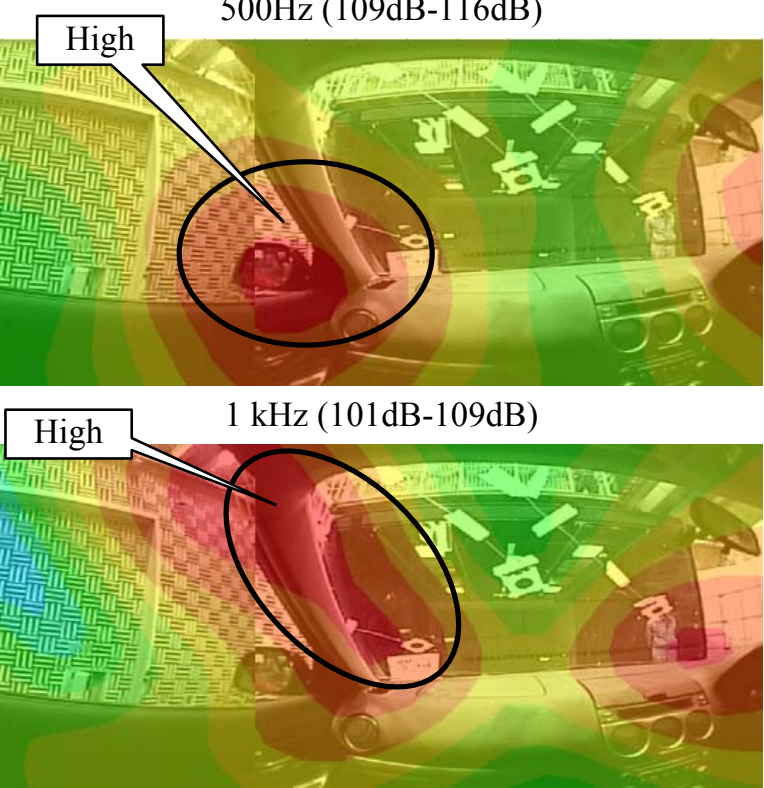

$2 \mathrm{kHz}(98 \mathrm{~dB}-104 \mathrm{~dB})$

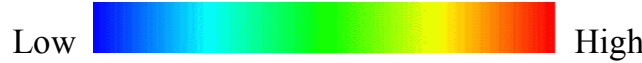

Fig.12 Internal wind noise sources measured by NoiseVision

Next, the relation between the outside and inside noise sources is described. Fig.13(a) shows the comparison of sound measured from the inside (Fig.11) and outside (Fig.7) of vehicle, and Fig.13(b) is a difference of the both. From Fig.13, we can see that the difference of sound measured from the outside and inside of vehicle increases as the frequency rises. This difference is regarded as the result of insulation structure of vehicle body. It indicates that the insulation performance of general vehicle body is strong in the high frequency range, and weak in the low frequency.

\section{Conclusions}

In this study, the structure of aerodynamic noise sources generated in a production vehicle has been investigated by wind tunnel experiment. The following conclusions are obtained.

(1) From the distribution of aerodynamic noise sources measured from the outside and inside of vehicle, it is found that the external noise source shows strong level in the regions of side window, bonnet head, roof head and front underbody around vehicle. The noise source in the front underbody is dominant in the low frequency range, and the noise source near side window becomes strong in the high frequency.

(2) By analyzing the velocity dependency of sound measured from the outside of vehicle, it is demonstrated that the main noise source is dipole induced by the pressure fluctuation on the surface of vehicle.

(3) The noise source generated near side window is induced by the flow separation in the front pillar and sideview mirror. Moreover, when the airflow to underbody is shut, the 
noise source in the underbody disappears, and the interior sound in the cabin of vehicle is significantly reduced.

(4) The location of noise source measured from the outside of vehicle is agreement with that measured from the inside of vehicle. However, since the insulation performance of vehicle body depends on the frequency, the aerodynamic noise in the low frequency is easy to transmit into the cabin in comparison with that of high frequency.

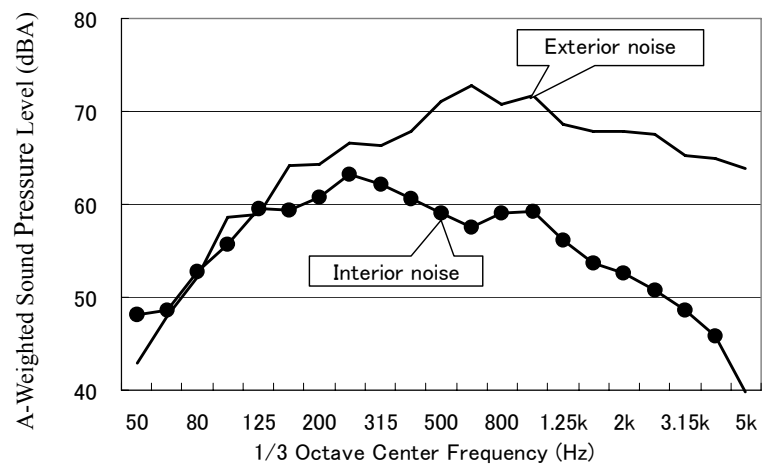

(a) Exterior and interior noise

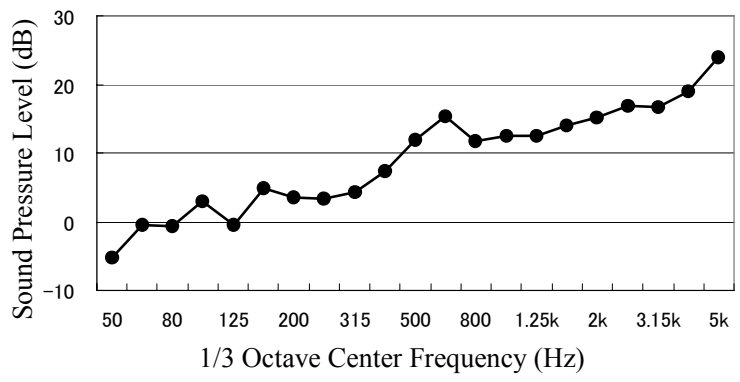

(b) Difference between exterior and interior noise

Fig.13 Comparison of exterior noise and interior noise

\section{References}

(1) Li Y., Kamioka T., Nouzawa T., Nakamura T., Okada Y. and Ichikawa N. , Evaluation of Aerodynamic Noise Generated in Production Vehicle Using Experiment and Numerical Simulation, Society of Automotive Engineers, 2003-01-1314 (2003).

(2) Alam F., Watkins S., Zimmer G. and Humphris C., Effects of Vehicle A-pillar Shape on Local Mean and Time-Varying Flow Properties, Society of Automotive Engineers, 2001-01-1086 (2001).

(3) Ih K.D., Lee J. and Kim Y., Development of Vehicle Underbody Acoustic Holography, Society of Automotive Engineers, 2002-01-1240 (2002).

(4) Christensen J.J., Hald J., Beamforming, Bruel \& Kjaer. TECHNICAL REVIEW, N0.1 (2004).

(5) Lighthill M. J., On sound generated aerodynamically: I. General theory, Proc. R. Soc. Lond. A 211 (1952), pp. 564-587.

(6) Curle N., The influence of solid boundaries upon aerodynamics sound. Proc. R. Soc. Lond. A 231 (1955), pp. 504-514. 